On the stability of Hamiltonian relative equilibria with non-trivial isotropy

Montaldi, James and Rodriguez-Olmos, Miguel 2011

MIMS EPrint: 2010.95

Manchester Institute for Mathematical Sciences

School of Mathematics

The University of Manchester

\footnotetext{
Reports available from: http://eprints.maths.manchester.ac.uk/

And by contacting: The MIMS Secretary

School of Mathematics

The University of Manchester

Manchester, M13 9PL, UK
} 


\title{
ON THE STABILITY OF HAMILTONIAN RELATIVE EQUILIBRIA WITH NON-TRIVIAL ISOTROPY
}

\author{
James Montaldi \& Miguel Rodríguez-Olmos
}

July 2011

Dedicated to the memory of Jerrold E. Marsden

\begin{abstract}
We consider Hamiltonian systems with symmetry, and relative equilibria with isotropy subgroup of positive dimension. The stability of such relative equilibria has been studied by Ortega and Ratiu [7] and by Lerman and Singer [3]. In both papers the authors give sufficient conditions for stability which require first determining a splitting of a subalgebra of $\mathfrak{g}$, with different splittings giving different criteria. In this note we remove this splitting construction and so provide a more general and more straightforward criterion for stability. The result is also extended to apply to systems whose momentum maps are not coadjoint equivariant.
\end{abstract}

Introduction Many Hamiltonian systems arising in nature possess symmetry and in particular continuous symmetry-most commonly a group of rotations or Euclidean motions, whether in the plane or in space. In this note we consider relative equilibria in such systems, which are motions that coincide with 1-parameter symmetry transformations. Given such a relative equilibrium, it is often important to decide on its (nonlinear) stability, and there are criteria for determining this based on Dirichlet's criterion for ordinary equilibria, but involving the velocity of the relative equilibrium through an appropriate element of the Lie algebra $\mathfrak{g}$ of the group $G$.

If the action is locally free at the relative equilibrium (meaning the isotropy subgroup at any point of the relative equilibrium is finite) then the "relative Dirichlet criterion" is straightforward because the velocity corresponds to a unique element of the Lie algebra $\mathfrak{g}$. However, when the action fails to be locally free the story is less clear because there will be many different "group velocities" for a given physical velocity. In the late 1990s two papers were published, by Ortega and Ratiu [7] and Lerman and Singer [3], adapting the Dirichlet criterion to deal with this case, while a paper by the first author [4] provides a more topological criterion, that of an "extremal relative equilibrium", which we will use in the proof below. The method of Ortega-Ratiu and Lerman-Singer involves having a splitting of the Lie algebra $\mathfrak{g}$, and showing there is a unique preferred group velocity relative to this splitting, which they call the 'orthogonal velocity', and then using this orthogonal velocity to define a relative Dirichlet criterion, analogous to the locally free case. Moreover, Ortega and Ratiu give an example showing how different choices of splitting may produce different critieria for stability so it may be necessary to consider all possible splittings.

The purpose of this note is to dispense with the splitting construction, and to show that the relative Dirichlet criterion is sufficient to guarantee stability, using any group veclocity, not merely those that arise from a splitting. In the special case that the relative equilibrium is an equilibrium, the orthogonal 
velocity is always zero, regardless of the splitting or inner product, and we give an example at the end of this note showing that it can be necessary to use a non-zero group velocity to establish the stability.

Since the proof is based on the idea of an extremal relative equilibrium introduced in [4], we need a technical assumption: that the momentum isotropy subgroup is compact, rather than it merely having a split subalgebra, as needed by Ortega-Ratiu and Lerman-Singer. Of course, if the group $G$ is compact then this is no loss of generality.

Setup and background Let $(P, \omega)$ be a connected symplectic manifold with a proper and Hamiltonian action of a Lie group $G$, with momentum map $\mathbf{J}: P \rightarrow \mathfrak{g}^{*}$ and an invariant Hamiltonian $h: P \rightarrow \mathbb{R}$. Recall that a momentum map is a map satisfying the differential condition

$$
\left\langle\mathrm{d} \mathbf{J}_{p}(\mathbf{v}), \xi\right\rangle=\omega\left(\xi_{P}(p), \mathbf{v}\right),
$$

for all $p \in P, \mathbf{v} \in T_{p} P, \xi \in \mathfrak{g}$, and is therefore uniquely determined up to a constant. By a theorem of Souriau [11] there is an action of $G$ on $\mathfrak{g}^{*}$ for which a given momentum map is equivariant, and any mention of isotropy groups and orbits of points in $\mathfrak{g}^{*}$ refers to this action. If $G$ is compact, the momentum map can be chosen so that this action is the coadjoint action $[4,8]$, but in general it requires an affine modification of the coadjoint action which we denote $\operatorname{Coad}_{g}^{\theta}$ :

$$
\operatorname{Coad}_{g}^{\theta} \mu=\operatorname{Coad}_{g} \mu+\theta(g),
$$

where $\theta: G \rightarrow \mathfrak{g}^{*}$ is a cocycle; details are in Souriau's book [11], see also [8].

Throughout, we will be referring to a point $p \in P$ and we write $H=G_{p}$, the isotropy subgroup at $p$ for the action on $P, \mu=\mathbf{J}(p)$ and $K=G_{\mu}$, the isotropy subgroup for the modified coadjoint action on $\mathfrak{g}^{*}$; in particular this means that $\mathbf{J}(k \cdot p)=\mu$ for all $k \in K$. We will also be assuming throughout that $K=G_{\mu}$ is compact, and this means that the momentum map can be chosen so that the cocycle $\theta$ vanishes on $K$, as pointed out in [5]. The Lie algebras of $H$ and $K$ are denoted $\mathfrak{h}$ and $\mathfrak{k}$ respectively.

A point $p \in P$ is a relative equilibrium if the Hamiltonian vector field at $p$ is tangent to the group orbit, or equivalently if $p$ is a critical point of the augmented Hamiltonian $h_{\xi}=h-\mathbf{J}_{\xi}$ for some $\xi \in \mathfrak{g}$, where $\mathbf{J}_{\xi}(x)=\langle\mathbf{J}(x), \xi\rangle$. We call such an element $\xi$ a group velocity of the relative equilibrium, to be contrasted with the actual/physical velocity in $T_{p} P$.

If $\mathfrak{h}$ is nonzero, then $\mathrm{d} \mathbf{J}_{\zeta}(p)=0$ for all $\zeta \in \mathfrak{h}$. Consequently, if $\xi$ is a group velocity at $p$ then so is $\xi+\zeta$. We begin with a lemma which is implicit in [7] (see their Theorem 2.8).

Lemma 1 Let $p \in P$ be a relative equilibrium, with $\mathbf{J}(p)=\mu$ and suppose $\xi \in \mathfrak{g}$ is a group velocity of $p$. Then $\xi \in \mathfrak{n}$, where $\mathfrak{n}:=\operatorname{Lie}\left(N_{K}(H)\right)$.

PROOF: Firstly, by conservation of momentum, $\xi \in \mathfrak{k}$, and secondly by conservation of symmetry $\xi \in \operatorname{Lie}\left(N_{G}(H)\right)$, the Lie algebra of the normalizer in $G$ of $H$. Combining these, one deduces that $\xi \in \mathfrak{n}$. Here 'conservation of symmetry' refers to the fact that if $x$ and $y$ lie in the same trajectory of a symmetric dynamical system, then their isotropy subgroups coincide: $G_{x}=G_{y}$.

We present some further details about the orthgonal velocities at the end of the paper.

A central ingredient in the stability analysis is the symplectic slice $N$ at a point $p \in P$, which is defined to be

$$
N:=\operatorname{kerd} \mathbf{J}(p) / \mathfrak{k} \cdot p .
$$

If the action in a neighbourhood of a relative equilibrium $p$ is locally free, so $\mathfrak{h}=0$, and if $K$ is compact, then there is a well-known criterion for assuring the stability of the relative equilibrium, 
extending Dirichlet's criterion for the Lyapounov stability of an equilibrium. The criterion is that the restriction $\left.\mathrm{d}^{2} h_{\xi}\right|_{N}$ of the Hessian to the symplectic slice should be definite. In particular, it was shown by Patrick [9] that under this assumption the relative equilibrium is Lyapounov stable relative to the subgroup $K$, which corresponds to the usual definition of Lyapounov stability but using $K$-invariant neighbourhoods. The situation where the action on $P$ is free but $K$ fails to be compact, and more generally where $\mu$ is not 'split', is considered in [10].

There are several results in the literature giving criteria for the stability of relative equilibria at points where the action is not locally free (so at points $p$ with $\mathfrak{h} \neq 0$ ). They all (as do we) require the group action on $P$ to be proper, at least in a neighbourhood of $p$. The criteria of Lerman-Singer [3] and Ortega-Ratiu [7] begin with requiring an $H$-invariant splitting of the Lie algebra $\mathfrak{k}$ in [3], or of $\mathfrak{n}$ in [7], as

$$
\mathfrak{k}=\mathfrak{m} \oplus \mathfrak{h} \quad \text { or } \quad \mathfrak{n}=\mathfrak{p} \oplus \mathfrak{h} .
$$

These decompositions are constructed by using an $H$-invariant inner product on $\mathfrak{k}$ or $\mathfrak{n}$, which exist as $H$ is compact, where $H$ acts on $\mathfrak{k}$ or $\mathfrak{n}$ by the adjoint action. The two cases are related by noting that $\mathfrak{n}$ is an invariant subspace under the action by $H$, so any invariant inner product on $\mathfrak{n}$ can be extended to one on $\mathfrak{k}$, while any one on $\mathfrak{k}$ restricts to one on $\mathfrak{n}$, and consequently one can choose $\mathfrak{p}=\mathfrak{m} \cap \mathfrak{n}$. If $\xi_{1} \in \mathfrak{g}$ is a group velocity of the relative equilibrium then $\xi_{1} \in \mathfrak{n}$, as pointed out in Lemma 1 above, and so the set of all velocities is the affine subspace $\xi_{1}+\mathfrak{h}$ of $\mathfrak{n}$.

The criterion for stability in both papers is as before that $\left.\mathrm{d}^{2} h_{\xi^{\perp}}\right|_{N}$ should be a definite quadratic form, where now $\xi^{\perp}$ is the orthogonal velocity which is defined to be the unique group velocity orthogonal to $\mathfrak{h}$ with respect to the chosen splitting, and hence contained in $\mathfrak{m}$ or $\mathfrak{p}$ (and hence always in $\mathfrak{p}$ ). Since the inner product (or splitting) is $H$-invariant, the uniqueness of $\xi^{\perp}$ shows that it is fixed by the adjoint action of $H$.

There is some flexibility in this construction as there may be a choice of invariant inner product, and usually a different choice of inner product leads to a different criterion. In particular if $\mathfrak{k}$ is Abelian, then any inner product is allowed, and hence any splitting, so if $p$ is not an equilibrium then any group velocity can be realized as an orthogonal velocity. Notice however, that if $p$ is an equilibrium then the orthogonal velocity is always 0 , regardless of the splitting (we give an explicit example at the end of this note). More details are in Proposition 5. Furthermore, it is not hard to find situations where there is a unique splitting in which case there is again a unique orthogonal velocity; for example if $G=G_{1} \times G_{2}$ with $G_{1}$ semisimple, $H=G_{1}$ and $\mu=0$, then $\xi^{\perp}=\left(0, \xi_{2}\right)$.

Main result The aim of this note is to eliminate the splitting construction, so to be able to use any group velocity for the criterion, not only orthogonal ones. We show below (Lemma 3 ) that for any group velocity $\xi$, the Hessian $\mathrm{d}^{2} h_{\xi}(p)$ induces a well-defined quadratic form on the symplectic slice, which we denote $\left.\mathrm{d}^{2} h_{\xi}\right|_{N}$. Note that in general this quadratic form is not $H$-invariant, although it is if $\xi$ is the orthogonal velocity for some splitting.

Theorem 2 Let $(P, \omega)$ be a symplectic manifold with a proper Hamiltonian action of the Lie group $G$, with momentum map $\mathbf{J}$, and let the smooth invariant function $h: P \rightarrow \mathbb{R}$ define a symmetric Hamiltonian system on $P$. Let $p$ be a relative equilibrium of this system, and suppose that $K:=G_{\mu}$ is compact, where $\mu=\mathbf{J}(p)$ and $G$ acts on $\mathfrak{g}^{*}$ so that $\mathbf{J}$ is equivariant. Let $\xi \in \mathfrak{g}$ be a group velocity of $p$. If the quadratic form $\left.\mathrm{d}^{2} h_{\xi}\right|_{N}$ on the symplectic slice at $p$ is definite then $p$ is Lyapounov stable relative to $K$.

Before proving this theorem, we recall the Witt-Artin decomposition of the tangent space $T_{p} P$ using the group action and symplectic form, and the Marle-Guillemin-Sternberg normal form. Define 
four sub-quotients of $T_{p} P$ as follows,

$$
\begin{aligned}
T_{0} & =\mathfrak{g} \cdot p \cap \operatorname{kerd} \mathbf{J}(p)=\mathfrak{k} \cdot p, \\
T & =\mathfrak{g} \cdot p / T_{0}, \\
N & =\operatorname{kerd} \mathbf{J}(p) / T_{0}, \\
N_{0} & =T_{p} M /(\mathfrak{g} \cdot p+\operatorname{kerd} \mathbf{J}(p)) .
\end{aligned}
$$

The subspaces $T$ and $N$ are symplectic, while $T_{0}$ is isotropic, and paired with $N_{0}$ by the symplectic form. The group action defines isomorphisms $\mathfrak{k} / \mathfrak{h} \simeq T_{0}$ and $\mathfrak{g} / \mathfrak{k} \simeq T$, so the symplectic form provides an isomorphism

$$
N_{0} \simeq(\mathfrak{k} / \mathfrak{h})^{*} \simeq \mathfrak{k}^{*} \cap \mathfrak{h}^{\circ} .
$$

Here $\mathfrak{k}^{*} \cap \mathfrak{h}^{\circ}$ is the annihilator of $\mathfrak{h}$ within $\mathfrak{k}^{*}$; some authors denote it by $\mathfrak{m}^{*}$ (after the splittings described above, though it is independent of the splitting).

Each space carries an action of the isotropy subgroup $H$, and as this group is compact there is an isomorphism of $H$-spaces

$$
T_{p} P \simeq T_{0} \oplus T \oplus N \oplus N_{0} .
$$

The vector space $N$ with its symplectic structure and action of $H$ is the symplectic slice and has its own homogeneous quadratic momentum map $\mathbf{J}_{N}: N \rightarrow \mathfrak{h}^{*}$.

The Witt-Artin decomposition determines the local geometry of the action, using the MarleGuillemin-Sternberg normal form. This states that there is an invariant neighbourhood of $p$ which is $G$-symplectomorphic to an invariant neighbourhood $U$ of $[e, 0,0]$ in the symplectic space $Y$ with momemtum map $\mathbf{J}_{Y}: Y \rightarrow \mathfrak{g}^{*}$ given by,

$$
\begin{aligned}
Y & =G \times_{H}\left(\left(\mathfrak{k}^{*} \cap \mathfrak{h}^{\circ}\right) \times N\right), \\
\mathbf{J}_{Y}([g, \rho, v]) & =\operatorname{Coad}_{g}^{\theta}\left(\mu+\rho+\mathbf{J}_{N}(v)\right) .
\end{aligned}
$$

Here the $H$-action on $G \times\left(\mathfrak{k}^{*} \cap \mathfrak{h}^{\circ}\right) \times N$ is by $h \cdot(g, \rho, v)=\left(\operatorname{gh}^{-1}, \operatorname{Coad}_{h} \rho, h \cdot v\right)$ (recall we have chosen $\theta$ to vanish on $K$, and hence on $H)$. The notation $[g, \rho, v]$ denotes the $H$-orbit of $(g, \rho, v)$. The $G$ action is simply $g_{1} \cdot[g, \rho, v]=\left[g_{1} g, \rho, v\right]$. Since a neighbourhood of $p$ in $P$ is diffeomorphic to $U \subset Y$, the Hamiltonian $h$ on $P$ defines a Hamiltonian on $U$, which we also denote by $h$.

ProOF OF THEOREM 2: The proof is in three stages. Firstly we apply the cross-section theorem of Gullemin and Sternberg [2], as modified in [5] to deal with non-coadjoint actions. This reduces the problem to a system on a smaller space with compact symmetry group $K$. Secondly, we show that the relative equilibrium is extremal in the sense of [4] and, since $K$ is compact, we deduce that is Lyapounov stable relative to $K$. And thirdly, we apply a result of Lerman and Singer [3] to deduce that the original relative equilibrium is also Lyapounov stable relative to $K$.

(1) Since $K$ is compact, there is a $K$-invariant slice $S_{\mu}$ to the modified coadjoint orbit $G \cdot \mu$. It is shown in [5, Section 3] that, in a neighbourhood of $p, R:=\mathbf{J}^{-1}\left(S_{\mu}\right)$ is a $K$-invariant symplectic submanifold of $P$, and that the momentum map $\mathbf{J}_{R}: R \rightarrow \mathfrak{k}^{*}$ can be chosen to be the full momentum map $\mathbf{J}$ restricted to $R$ followed by the natural projection $\mathfrak{g}^{*} \rightarrow \mathfrak{k}^{*}$. Furthermore, as $R$ is a union of level sets of $\mathbf{J}$, it is invariant under the original dynamics, so the restriction of $h$ to $R$ determines the dynamics on $R$ by the usual equations of Hamilton. Note that the Witt-Artin decomposition of $T_{p} R$ is

$$
T_{p} R=T_{0} \oplus N \oplus N_{0}
$$

where $T_{0}, N$ and $N_{0}$ are the same spaces as in eq. (3), and $\operatorname{ker} \mathrm{d} \mathbf{J}_{R}(p)=T_{0} \oplus N$. 
(2) We want to show that the relative equilibrium $p$ is extremal [4]; this means that the corresponding equilibrium point in the reduced space is a local extremum of the reduced Hamiltonian. Let us assume that $\left.\mathrm{d}^{2} h_{\xi}\right|_{N}$ is positive definite (it is well-defined by Lemma 3 below); the negative definite case proceeds similarly. We therefore want to show there is a neighbourhood $U$ of $p$ in $\mathbf{J}^{-1}(\mu)=\mathbf{J}_{R}^{-1}(\mu)$ such that $x \in U \backslash K \cdot p \Rightarrow h(x)>h(p)$.

To this end we use the Marle-Guillemin-Sternberg normal form (4), with $G$ replaced by $K$, thus:

$$
\begin{aligned}
Z & =K \times_{H}\left(\mathfrak{h}^{\circ} \times N\right), \\
\mathbf{J}_{Z}([g, \rho, v]) & =\mu+\operatorname{Coad}_{g}\left(\rho+\mathbf{J}_{N}(v)\right),
\end{aligned}
$$

where $\mathbf{J}_{Z}: Z \rightarrow \mathfrak{k}^{*}$. (Since $g \in K$ we have $\operatorname{Coad}_{g}(\mu)=\mu$, and we now take $\mathfrak{h}^{\circ}$ to mean the annihilator of $\mathfrak{h}$ inside $\mathfrak{k}^{*}$.)

From eq. (5), one sees that $\mathbf{J}_{Z}^{-1}(\mu)=\left\{[g, \rho, v] \in Z \mid \rho=0, \mathbf{J}_{N}(v)=0\right\}$ (this can also be found in [1, Proposition 13]), so that the reduced space at $\mu$ in this model is

$$
Z_{\mu}=\mathbf{J}_{Z}^{-1}(\mu) / K \simeq \mathbf{J}_{N}^{-1}(0) / H .
$$

Now let $\bar{q} \in Z_{\mu}$ be a point distinct from $\bar{p}$, and let $q \in N$ be a corresponding point in $\mathbf{J}_{N}^{-1}(0)$. We wish to show $h_{\xi}(q)>h_{\xi}(p)$.

We are assuming $\mathrm{d}^{2} h_{\xi}$ to be non-degenerate. By the Morse Lemma there is therefore a diffeomorphism $\phi$ of $N$ (preserving $p$ ) such that $h_{\xi}=h_{\xi}(p)+\left.\mathrm{d}^{2} h_{\xi}\right|_{N} \circ \phi$. It follows, using the fact that $\left.\mathrm{d}^{2} h_{\xi}\right|_{N}$ is positive definite, that $h_{\xi}(q)=h_{\xi}(p)+\left.\mathrm{d}^{2} h_{\xi}\right|_{N}(\phi(q))>h_{\xi}(p)$, as required.

(3) Finally, Proposition 2.3 of Lerman and Singer [3] states that if $p$ is a relative equilibrium on $R$ which is Lyapounov stable relative to $K$, then $p$ is also Lyapounov stable relative to $K$ in the full $G$-invariant system on $P$.

The theorem is proved modulo the lemma below, required for part (2) of the proof.

Lemma 3 If $p$ is a relative equilibrium and $\xi \in \mathfrak{g}$ is a group velocity, then the restriction of the quadratic form $\mathrm{d}^{2} h_{\xi}(p)$ to ker $\mathrm{d} \mathbf{J}$ descends to a well-defined quadratic form on the symplectic slice $N$.

PROOF: Since the Hamiltonian vector field is equivariant, the point $g \cdot p$ is a relative equilibrium with group velocity $\operatorname{Ad}_{g} \xi$, and hence for all $g \in G$, the corresponding differential vanishes:

$$
\mathrm{d} h_{\mathrm{Ad}_{g} \xi}(g \cdot p)=0 .
$$

Write $g=\exp (t \eta)$ for $\eta \in \mathfrak{g}$, and differentiate with respect to $t$ at $t=0$ to obtain

$$
\mathrm{d}^{2} h_{\xi}(\eta \cdot p,-)-\mathrm{d} \mathbf{J}_{[\eta, \xi]}=0,
$$

where the differentials are taken at $p$. It follows that for any $v \in \operatorname{kerd} \mathbf{J}(p)$ we have

$$
\mathrm{d}^{2} h_{\xi}(\eta \cdot p, v)=0
$$

It then follows that, given any $\eta \in \mathfrak{k}$ (so that $\eta \cdot p \in \operatorname{ker} \mathrm{d} \mathbf{J}(p)$ )

$$
\mathrm{d}^{2} h_{\xi}(v+\eta \cdot p, v+\eta \cdot p)=\mathrm{d}^{2} h_{\xi}(v, v)
$$

as required. 
Example 4 Let $P=S^{2} \times S^{2} \times S^{2}$ with symplectic form $\omega=\omega_{0} \oplus \omega_{0} \oplus-\omega_{0}$, where $\omega_{0}$ is the standard symplectic form on $S^{2}$. The group $G=\mathrm{SO}(3)$ acts diagonally on $P$, and has momentum map $\mathbf{J}\left(\mathbf{x}_{1}, \mathbf{x}_{2}, \mathbf{x}_{3}\right)=\mathbf{x}_{1}+\mathbf{x}_{2}-\mathbf{x}_{3}$ (after identifying $\mathfrak{s o}(3)^{*}$ with $\left.\mathbb{R}^{3}\right)$. The point $p=\left(\mathbf{e}_{3}, \mathbf{e}_{3}, \mathbf{e}_{3}\right) \in P$ (the North poles) has isotropy subgroup $G_{p}=\mathrm{SO}(2)$, given by rotations about the $z$-axis, and momentum value $\mu=\mathbf{J}(p)=\mathbf{e}_{3}$. The momentum isotropy subgroup satisfies $G_{\mu}=G_{p}$ so $\mathfrak{k}=0$ and

$$
N=\operatorname{kerd} \mathbf{J}_{p}=\left\{\left(\hat{\mathbf{x}}_{1}, \hat{\mathbf{x}}_{2}, \hat{\mathbf{x}}_{3}\right) \in T_{p} P \mid \hat{\mathbf{x}}_{1}+\hat{\mathbf{x}}_{2}-\hat{\mathbf{x}}_{3}=0\right\} .
$$

Now, $p$ is necessarily an equilibrium point for any $G$-invariant Hamiltonian (for example, it is isolated in $\operatorname{Fix}(\mathrm{SO}(2), P))$. Now consider the Hamiltonian $h\left(\mathbf{x}_{1}, \mathbf{x}_{2}, \mathbf{x}_{3}\right)=\mathbf{x}_{2} \cdot \mathbf{x}_{3}+\mathbf{x}_{3} \cdot \mathbf{x}_{1}-2 \mathbf{x}_{1} \cdot \mathbf{x}_{2}$, which is $G$-invariant. Writing $\hat{\mathbf{x}}_{1}=(a, b, 0)$ and $\hat{\mathbf{x}}_{2}=(c, d, 0)$ we have $\hat{\mathbf{x}}_{3}=(a+c, b+d, 0)$, and the Hessian in the coordinates $(a, c, b, d)$ turns out to be

$$
\left[\mathrm{d}^{2} h-\mathrm{d}^{2} \mathbf{J}_{\xi}\right]_{p}=\left(\begin{array}{cccc}
1 & -2-\xi & 0 & 0 \\
-2-\xi & 1 & 0 & 0 \\
0 & 0 & 1 & -2-\xi \\
0 & 0 & -2-\xi & 1
\end{array}\right)
$$

This is positive definite for $\xi \in(-3,-1)$, and so by Theorem 2 the equilibrium point $p$ is Lyapounov stable relative to $\mathrm{SO}(2)$ (which in fact means it is Lyapunov stable). However, as explained earlier, the criteria of Lerman-Singer and Ortega-Ratiu do not guarantee stability as the unique orthogonal velocity is 0 and $d^{2} h(0)$ is not definite.

A different proof of Theorem 2, using the so-called bundle equations, will be given in [6], with a different example.

Orthogonal velocities Although we do not need this for the main result, we present a characterisation of those group velocities which can be orthogonal velocities for some suitably chosen invariant splitting; this is helpful for comparing our theorem with those of Ortega-Ratiu and Lerman-Singer.

Proposition 5 (1) For any $H$-invariant splitting, the orthogonal velocity is in $\mathfrak{p}^{H}$.

(2) If $p$ is an equilibrium, then $\xi^{\perp}=0$ for any splitting.

(3) If $p$ is a relative equilibrium but not an equilibrium, and $\xi_{0}$ is any group velocity fixed by $H$ under the adjoint action, then there is an $H$-invariant splitting for which $\xi^{\perp}=\xi_{0}$.

Parts (1) and (3) show that for a relative equilibrium which is not an equilibrium, a group velocity is an orthogonal velocity for some invariant splitting if and only if it is fixed by the adjoint action of $H$.

PROOF: (1) is proved above (it follows from the uniqueness of $\xi^{\perp}$ ), (2) is obvious and (3) requires a little more analysis. Refine a given invariant splitting $\mathfrak{n}=\mathfrak{h} \oplus \mathfrak{p}$ (see Equation (2) above) into the $H$-fixed parts and their invariant complements (under the adjoint action of $H$ on $\mathfrak{n}$ ):

$$
\mathfrak{n}=\left(\mathfrak{h}^{H} \oplus \mathfrak{h}^{\prime}\right) \oplus\left(\mathfrak{p}^{H} \oplus \mathfrak{p}^{\prime}\right) .
$$

By hypothesis, $\xi_{0} \in \mathfrak{h}^{H} \oplus \mathfrak{p}^{H}$. Now on the subspace $\mathfrak{h}^{H} \oplus \mathfrak{p}^{H}$ the action of $H$ is trivial, so any quadratic form is invariant. Since $\xi_{0} \notin \mathfrak{h}^{H}$ we can choose an inner product on this subspace so that $\xi_{0}$ is orthogonal to $\mathfrak{h}^{H}$. Now extend this chosen inner product to an invariant one on $\mathfrak{n}$ and we still have $\xi_{0} \in\left(\mathfrak{h}^{H}\right)^{\perp}$. Since the inner product is invariant one has moreover that $\xi_{0} \in\left(\mathfrak{h}^{\prime}\right)^{\perp}$ so that $\xi_{0} \in \mathfrak{h}^{\perp}$ as required. 
Acknowledgements The research of M.R-O. was supported by the research project MTM200603322 and a European Marie Curie Fellowship (IEF) held at the University of Manchester. We also thank the referees for useful comments.

\title{
References
}

[1] L. Bates and E. Lerman, Proper group actions and symplectic stratified spaces. Pacific J. Math. 181 (1997), 201-229.

[2] V. Guillemin and S. Sternberg, Symplectic Techniques in Physics. Cambridge University Press, 1984.

[3] E. Lerman and S. Singer, Relative equilibria at singular points of the momentum map. Nonlinearity 11 (1998), 1637-1649.

[4] J. Montaldi, Persistence and stability of relative equilibria. Nonlinearity 10 (1997), 449-466.

[5] J. Montaldi \& T. Tokieda, Openness of momentum maps and persistence of extremal relative equilibria. Topology 42 (2003), 833-844.

[6] J. Montaldi and M. Rodríguez-Olmos, Hamiltonian relative equilibria with continuous isotropy. In preparation.

[7] J-P. Ortega and T.S. Ratiu, Stability of Hamiltonian relative equilibria. Nonlinearity 12 (1999), 693-720.

[8] J-P. Ortega and T.S. Ratiu, Momentum Maps and Hamiltonian Reduction. Birkhäuser, 2004.

[9] G.W. Patrick, Relative equilibria in Hamiltonian systems: the dynamic interpretation of nonlinear stability on a reduced phase space. J. Geo. Phys. 9 (1992), 111-119.

[10] G.W. Patrick, R.M. Roberts \& C. Wulff, Stability of Poisson Equilibria and Hamiltonian Relative Equilibria by Energy Methods. Arch. Rational Mech. Anal. 174 (2004) 301-344.

[11] J.-M. Souriau, Structure des Systèmes Dynamiques, Dunod, Paris, 1970. (English translation: Structure of Dynamical Systems, Birkhauser, Boston, 1997).

\author{
School of Mathematics \\ University of Manchester \\ Manchester M13 9PL \\ $U K$ \\ Department of Applied Mathematics IV \\ Technical University of Catalonia \\ Barcelona \\ Spain
}

